\title{
Modification of the Female Figure Identification Technique (FFIT) Formulas to Include Plus Size Bodies
}

\author{
Susan L. SOKOLOWSKI *, Chrissy BETTENCOURT \\ Sports Product Design, University of Oregon, Portland (OR), USA \\ https://doi.org/10.15221/20.22
}

\begin{abstract}
With new 3D plus size body scan data available through surveys like Size North America and manufacturers investing in their own data, there was an opportunity to study the shape of modern female plus sized bodies to inform the fit of products for this emerging business demographic. The researchers partnered with a leading apparel company to analyze 3D plus size body scans with the Female Figure Identification Technique (FFIT) for apparel developed by Simmons, Istook, and Devarajan (2004), using mathematical representations of the FFIT body shapes created by Lee, Istook, Nam, and Park (2007). However, during the project, it was discovered through visual inspections there were opportunities to modify the FFIT mathematical formulas to be more inclusive of plus size women. The inspections indicated that some scans were inaccurately classified or not sorted into any shape category. Since plus size women often have larger abdomens than bust or hips, the formulas were modified to include a check for that condition. By understanding shape, manufacturers can have a better idea of how to design, fit and grade products for this market throughout a size range, as opposed to relying on only $2 \mathrm{D}$ measurements or linear grading rules from a sample size.
\end{abstract}

Keywords: 3D body scanning, plus sizes, Female Figure Identification Technique (FFIT) for apparel

\section{Introduction}

In 2017, the Centers for Disease Control and Prevention reported the prevalence of adult obesity at $39.8 \%$ of the United States (U.S.) population, which affects about 93.3 million [1]. Of those adults, $41 \%$ were estimated to be women [1]. With new 3D plus size body scan data available through surveys like Size North America and manufacturers investing in their own content, there was an opportunity to study the shape of modern female plus sized bodies. By understanding shape, manufacturers can have a better idea of how to design, fit and grade products throughout a size range, as opposed to relying on only 2D measurements or linearly grading from a sample size that could be as small as a U.S. size 4/6. Shape provides better context of how 2D measures are distributed around a body, how design considerations like materials, adjustment features and patterns should be integrated, and if linear grades are relevant.

For this study the researchers partnered with a leading apparel company to analyze 3D plus size body scans using the Female Figure Identification Technique (FFIT) for apparel [2,3]. However, during the course of the research it was discovered there were opportunities to modify the FFIT formulas to better include plus size 3D scans. This paper will review current challenges with the U.S. plus sizing, the history of the FFIT for apparel software, limitations discovered when evaluating plus size scans with the technique and modifications to FFIT formulas to be more inclusive of plus sizes.

\section{Background}

\subsection{U.S. plus size apparel industry}

In the U.S., the female plus sized demographic represents around a $\$ 21.4$ billion apparel industry [4]. Over the last five years, there has been an increased interest from manufacturers to develop products for this body type [4]. As a result of a "speed to market behavior," previous research by Sokolowski, Griffin and Silbert found that preferred U.S. retailers are inconsistently and unreliably sizing plus apparel [5]. Tables 1-6 demonstrate those inconsistencies and how the measures assume an Hourglass shape. These challenges not only affect consumer satisfaction, but also sell-through and the environmental sustainability due to excessive product returns from poor fit [5].

\footnotetext{
* ssokolow@uoregon.edu; +1-503-799-6634; https://artdesign.uoregon.edu/pd/graduate
} 
Table 1. Walmart plus size measures.

\begin{tabular}{llll}
\hline Size & Bust & Waist & Hip \\
\hline $16 \mathrm{~W}$ & $42.5 "$ & $34.5 "$ & $44.5^{\prime \prime}$ \\
$18 \mathrm{~W}$ & $44.5 "$ & $36.5 "$ & $46.5 "$ \\
$20 \mathrm{~W}$ & $46.5 "$ & $38.5 "$ & $48.5 "$ \\
$22 \mathrm{~W}$ & $48.5 "$ & $40.5 "$ & $50.5 "$ \\
$24 \mathrm{~W}$ & $50.5 "$ & $42.5 "$ & $52.5 "$ \\
$26 \mathrm{~W}$ & $52.5 "$ & $44.5 "$ & $54.5 "$ \\
$28 \mathrm{~W}$ & $54.5 "$ & $46.5 "$ & $56.5 "$ \\
$30 \mathrm{~W}$ & $56.5 "$ & $48.5 "$ & $58.5 "$ \\
$32 \mathrm{~W}$ & $58.5 "$ & $50.5 "$ & $60.5 "$ \\
\hline
\end{tabular}

Table 3. JC Penney plus size measures.

\begin{tabular}{llll}
\hline Size & Bust & Waist & Hip \\
\hline $14 \mathrm{~W}$ & $42 "$ & $36 "$ & $45^{\prime \prime}$ \\
$16 \mathrm{~W}$ & $44 "$ & $38 "$ & $47 "$ \\
$18 \mathrm{~W}$ & $46 "$ & $40 "$ & $49 "$ \\
$20 \mathrm{~W}$ & $48 "$ & $42 "$ & $51 "$ \\
$22 \mathrm{~W}$ & $50 "$ & $44 "$ & $53 "$ \\
$24 \mathrm{~W}$ & $52 "$ & $46 "$ & $55^{\prime \prime}$ \\
$26 \mathrm{~W}$ & $54 "$ & $48 "$ & $57 "$ \\
$28 \mathrm{~W}$ & $56 "$ & $50 "$ & $59 "$ \\
$30 \mathrm{~W}$ & $58 "$ & $52 "$ & $61 "$ \\
$32 \mathrm{~W}$ & $60 "$ & $54 "$ & $63 "$ \\
$34 \mathrm{~W}$ & $62 "$ & $56 "$ & $65 "$ \\
\hline
\end{tabular}

Table 5. Macy's (Michael Kors) plus size measures.

\begin{tabular}{llll}
\hline Size & Bust & Waist & Hip \\
\hline $14 \mathrm{~W}$ & $43.5 "$ & $35 "$ & $44^{\prime \prime}$ \\
$16 \mathrm{~W}$ & $45.5 "$ & $37 "$ & $46 "$ \\
$18 \mathrm{~W}$ & $47.5 "$ & $39 "$ & $48 "$ \\
$20 \mathrm{~W}$ & $49.5 "$ & $41 "$ & $50 "$ \\
$22 \mathrm{~W}$ & $51.5 "$ & $43 "$ & $52 "$ \\
$24 \mathrm{~W}$ & $53.5 "$ & $45 "$ & $54 "$ \\
\hline
\end{tabular}

Table 2. Kohl's (Chaps) plus size measures.

\begin{tabular}{llll}
\hline Size & Bust & Waist & Hip \\
\hline $14 \mathrm{~W}$ & $42 "$ & $37 "$ & $45^{\prime \prime}$ \\
$16 \mathrm{~W}$ & $44 "$ & $39 "$ & $47 "$ \\
$18 \mathrm{~W}$ & $46 "$ & $41 "$ & $49 "$ \\
$20 \mathrm{~W}$ & $48 "$ & $43 "$ & $51 "$ \\
$22 \mathrm{~W}$ & $50 "$ & $45 "$ & $53 "$ \\
$24 \mathrm{~W}$ & $52 "$ & $47 "$ & $55 "$ \\
$26 \mathrm{~W}$ & $54 "$ & $49 "$ & $57 "$ \\
\hline
\end{tabular}

Table 4. Target (Wild Fable) plus size measures.

\begin{tabular}{|c|c|c|c|}
\hline Size & Bust & Waist & Hip \\
\hline $14 \mathrm{~W}$ & $41 "$ & $36 "$ & $43^{\prime \prime}$ \\
\hline $16 \mathrm{~W}$ & $43 "$ & $38 "$ & $45^{\prime \prime}$ \\
\hline $18 \mathrm{~W}$ & $45 "$ & $40 "$ & $47^{\prime \prime}$ \\
\hline $20 \mathrm{~W}$ & $47 "$ & $42.25 "$ & $49 "$ \\
\hline $22 \mathrm{~W}$ & $49 "$ & 44.5” & $51 "$ \\
\hline $24 \mathrm{~W}$ & 51" & $46.75 "$ & $53 "$ \\
\hline $26 \mathrm{~W}$ & $53 "$ & $49 "$ & $55^{\prime \prime}$ \\
\hline $28 \mathrm{~W}$ & $55 "$ & $51.25 "$ & $57 "$ \\
\hline $30 \mathrm{~W}$ & 57" & 53.5” & $59 "$ \\
\hline
\end{tabular}

Table 6. Lane Bryant plus size measures.

\begin{tabular}{|c|c|c|c|}
\hline Size & Bust & Waist & Hip \\
\hline 14 & $42 "$ & $36 "$ & $44.5 "$ \\
\hline 16 & $44 "$ & $38 "$ & $46.5 "$ \\
\hline 18 & $46 "$ & $40 "$ & 48.5" \\
\hline 20 & $48 ”$ & 42” & $50.5 "$ \\
\hline 22 & $50 "$ & $44^{\prime \prime}$ & $52.5^{\prime \prime}$ \\
\hline 24 & $52 "$ & $46 "$ & 54.5" \\
\hline 26 & $54 "$ & $48 "$ & $56.5^{\prime \prime}$ \\
\hline 28 & $56 "$ & $50 "$ & $58.5 "$ \\
\hline 30 & $58 "$ & $52 "$ & $60.5 "$ \\
\hline 32 & $60 "$ & $54 "$ & $62.5^{\prime \prime}$ \\
\hline
\end{tabular}

\subsection{Body shape as a tool for apparel design, fit and sizing}

In the same study discussed in section 2.1., 65 size 18 female body scans were matched to the size charts presented in Tables 1-6. It was found that $98.46 \%$ of the scans did not fit into any of the charts [5]. In another plus size study conducted by Sokolowski and Bettencourt, it was found that none of the body scans studied were an Hourglass or Top Hourglass shape and only a small percentage $(8.7 \%)$ were a Bottom Hourglass shape [6]. The findings from both studies demonstrated that apparel manufacturers should 1) refrain from linearly grading products from smaller Hourglass shapes and 2) consider shape of body to understand how measures are distributed around a body, to inform not only sizing but design details (e.g., material placement, pattern shape, adjustment features).

\subsection{Female Figure Identification Technique (FFIT) for Apparel}

In 2004, Simmons, Istook, and Devarajan developed a shape sorting software, called the Female Figure Identification Technique (FFIT) for apparel, to classify 3D body scans and identify body shapes [2,3]. The software uses anthropometric measurements captured from 3D body scans to sort subjects into one of nine shape categories: Hourglass, Top Hourglass, Bottom Hourglass, Spoon, Rectangle, Diamond, Oval, Triangle, and Inverted Triangle. Bust, waist, and hip circumferences were used to determine which body shape the scan matched to. Stomach and abdomen circumferences were also used to determine Diamond and Oval shapes; however, Simmons et al. did not provide detailed descriptions of where on the scan these measurements were taken. Definitions of the shapes include: 
1. Hourglass: proportional bust and hip measurements, with a defined waistline.

2. Bottom Hourglass: larger hip than bust, with a defined waistline.

3. Top Hourglass: larger bust than hip, with a defined waistline.

4. Spoon: a significant difference between hip and bust, a bust-to-waist ratio smaller than Hourglass, and a significant high hip-to-waist ratio, indicating a "shelf" where the waist drops off sharply with similar hip and high hip measurements.

5. Triangle: a larger hip than bust without a defined waist.

6. Inverted Triangle: a larger bust than hip without a defined waist.

7. Rectangle: similar bust and hip measurements, without a defined waistline.

8. Diamond: a very large midsection with the average of stomach, waist, and abdomen larger than bust.

9. Oval: a large midsection with the average of stomach, waist, and abdomen smaller than bust.

\subsection{FFIT for apparel mathematical formulas}

In a 2007 study by Lee, Istook, Nam, and Park, body shapes of women from the U.S. were compared to those from Korea, using measurement data from SizeUSA and SizeKorea with the FFIT for Apparel system [7]. In order to determine the efficacy of sorting USA and Korean women, Lee et al. used a mathematical analysis and visual inspection to develop formulas based on the descriptions of the original FFIT software categories (Table 7). The formulas used bust, hip, waist, and high hip measurements to match body measures into one the following shape categories: Hourglass, Bottom Hourglass, Top Hourglass, Spoon, Triangle, Inverted Triangle, or Rectangle [7]. While Simmons et al. initially included nine formulas, Lee et al. did not include Diamond or Oval in their formulas. Although not documented, the researchers believe this was likely due to lack of measurement data (stomach and abdomen measures, which were undefined) and larger body types.

Table 7. Original Female Figure Identification Technique (FFIT) formulas (in inches) [7].

\begin{tabular}{|l|l|}
\hline BODY TYPE... & MEASUREMENT \\
\hline Hourglass & If (bust-hip) $\leq 1$, then if (hip-bust) $<3.6$, then if (bust-waist) $\geq 9$ or (hip-waist) $\geq 10$ \\
\hline $\begin{array}{l}\text { Bottom } \\
\text { Hourglass }\end{array}$ & If (hip-bust) $\geq 3.6$ and (hip-bust) $<10$, then if (hip-waist) $\geq 9$, then if (high hip/waist) $<1.193$ \\
\hline Top Hourglass & If (bust-hip) $>1$ and (bust-hip) $<10$, then if (bust-waist) $\geq 9$ \\
\hline Spoon & If (hip-bust) $>2$, then if (hip-waist) $\geq 7$, then if (high hip/waist) $\geq 1.193$ \\
\hline Triangle & If (hip-bust) $\geq 3.6$, then if (hip-waist) $<9$ \\
\hline Inverted Triangle & If (bust-hip) $\geq 3.6$, then if (bust-waist) $<9$ \\
\hline Rectangle & If (hip-bust) $<3.6$, and (bust-hip) $<3.6$, then if (bust-waist) $<9$ and (hip-waist) $<10$ \\
\hline
\end{tabular}

\subsection{Challenges with 2007 FFIT formulas}

For this study, the 2007 FFIT formulas were used to sort plus size 3D scans. The formulas were successful in classifying many of the scans; however, discrepancies were discovered between the body shapes defined by the formulas and the visually identifiable shapes. Many scans were classified as Rectangles or Inverted Triangles based on the mathematical formulas, but visual inspection indicated that these categories were not accurate, often because the waist circumferences were larger than either bust or hip. In addition, some scans did not fit into any of the shape definitions. In all, it was found that the existing 2007 FFIT formulas had an underlying assumption that the waist was smaller than the bust and hip.

\section{Experimental Procedures}

\subsection{Modifications to the FFIT formulas for plus size scans}

In order to correct the discrepancies discovered with the 2007 FFIT formulas, the 3D plus scans analyzed for this study were grouped into size categories $16,18,20,24$, and 28 , based on both bust and hip circumferences, resulting in 10 different size groups.

The Hourglass, Bottom Hourglass, Top Hourglass, and Spoon shape formulas were left unchanged. These shape categories were not common among plus size scans, and in fact, none of them were classified as an Hourglass. Furthermore, these categories required a defined waist, with a difference between the bust and waist measurements (bust-waist) exceeding 9" for the Hourglass and Top Hourglass shapes, and a difference between the hip and waist measurements (hip-waist) exceeding 9" 
or 7" for Bottom Hourglass and Spoon, respectively. Most of the scans analyzed were significantly less than or often negative measures, indicating a large abdomen compared to the bust and/or hip. Visual inspection of scans in these categories also confirmed that they were accurately sorted. The result was that these formulas were sufficient to rule out any misplaced Diamond or Oval body shapes.

The Triangle, Inverted Triangle, and Rectangle shapes, however, were inaccurately applied to many plus size scans with large abdomens, and so modifications were needed to accurately sort these scans. The measurements listed in Table 8 illustrate how three size 16 subjects' scans were categorized as an Inverted Triangle or Rectangle. Visual inspection confirmed that these scans were inaccurately sorted, and examples of this challenge are presented in Fig. 1.

Table 8. Measurements of size 16 subjects' scans that were incorrectly identified.

\begin{tabular}{|l|l|l|l|l|l|l|}
\hline SUB & $\begin{array}{l}\text { BUST- } \\
\text { HIP }\end{array}$ & $\begin{array}{l}\text { HIP- } \\
\text { BUST }\end{array}$ & $\begin{array}{l}\text { BUST- } \\
\text { WAIST }\end{array}$ & $\begin{array}{l}\text { HIP- } \\
\text { WAIST }\end{array}$ & $\begin{array}{l}\text { HIGH HIP- } \\
\text { WAIST }\end{array}$ & $\begin{array}{l}\text { INITIAL BODY } \\
\text { SHAPE }\end{array}$ \\
\hline$\# 1$ & $4.88 "$ & $-4.88 "$ & $4.17 "$ & $-0.71 "$ & 1.07 & Inverted Triangle \\
\hline$\# 2$ & $-1.81 "$ & $1.81 "$ & $-3.90 "$ & $-2.09 "$ & 0.99 & Rectangle \\
\hline$\# 3$ & $-2.95 "$ & $2.95 "$ & $-2.83 "$ & $0.12 "$ & 1.05 & Rectangle \\
\hline
\end{tabular}
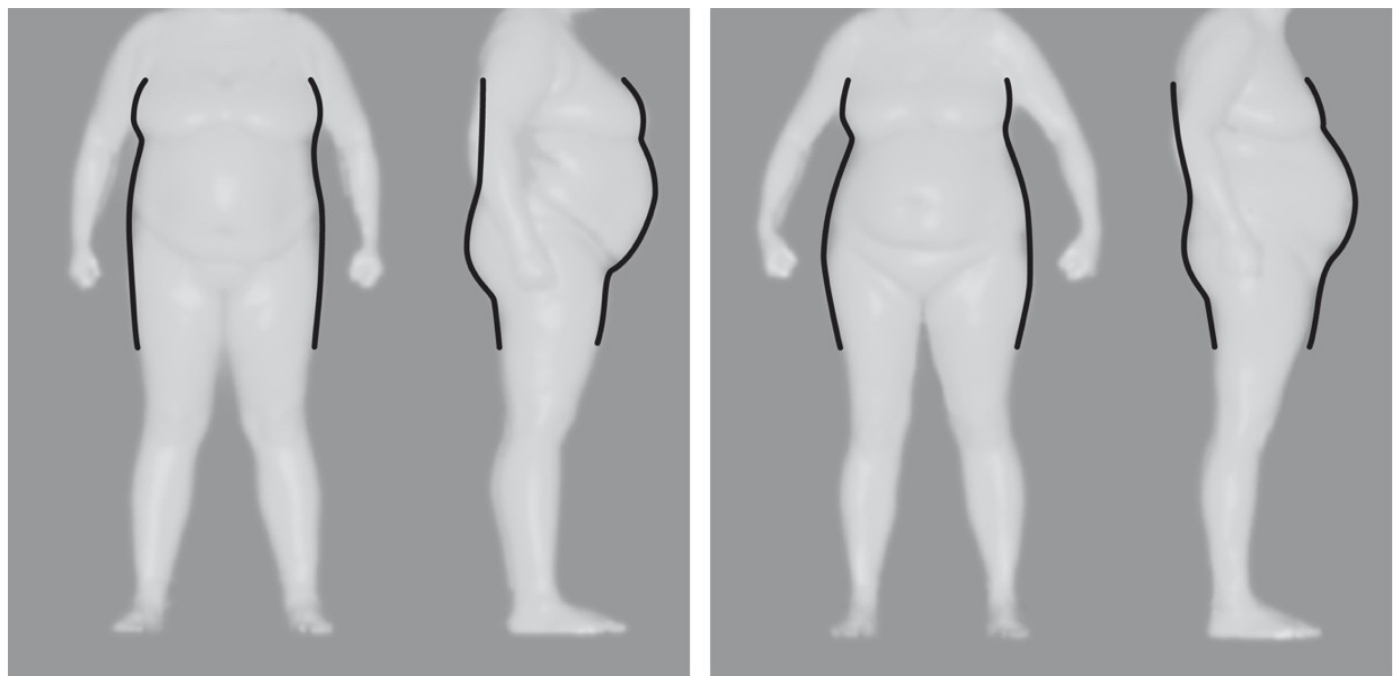

Fig. 1. Subject \#1 (left) and Subject \#2 (right), inaccurately categorized as Inverted Triangle and Rectangle shapes, respectively. Visual inspection indicated that they should have been categorized as Oval or Diamond shapes due to large abdomens.

For subjects \#1 and \#2 in Table 8, the negative difference between hip and bust measurements (hipbust) indicated that the abdomens of the scans were larger than hips. The formulas have an underlying assumption that the waist is always smaller than the bust and hip, as was the case with most scans under size 14 analyzed in previous studies $[2,3,7]$. In order to correct for the assumption that the waist was smaller than the bust or hip, a check for negative numbers was added to the Triangle, Rectangle, and Inverted Triangle calculations. For the Triangle shape, the formula (hip-waist) $<9$ was changed to $0 \leq$ (hip-waist) $<9$, which accurately removed any scan with a defined waist as well as those with a very large abdomen. For the Inverted Triangle shape, a separate calculation was added, (hip-waist) $\geq 0$, after it was determined that the bust was significantly larger than the hip. Similar to the Triangle shape, for the Rectangle shape the formula (hip-waist) $<10$ was changed to $0 \leq$ (hip-waist) $<10$, to accurately remove any scan with a larger abdomen.

The two missing categories, Oval and Diamond, were reintroduced to accurately sort scans with larger waist measurements than hips. According to Simmons et al. [3], the distinction between a Diamond and an Oval shape was determined by the abdomen compared to the bust measurement. After the scans were determined to fall outside of the values of the Rectangle, Triangle, and Inverted Triangle shapes as indicated by a negative difference between hip and waist measurements, (hip-waist) a positive difference between bust and waist measurements (bust-waist) indicated an Oval shape, and a negative value indicated a Diamond shape. 
Another instance in which the 2007 FFIT formulas failed to correctly categorize plus size scans was with the Triangle shape. In order for a scan to be considered a Triangle shape, it must have met the criteria that the hip measurement was significantly larger than the bust. In Table 8, Subject \#3's scan was classified as a Rectangle because the hip circumference was not significantly greater, 3.6" or more, than the bust circumference. However, the negative difference between bust and waist measurements (bust-waist) and positive difference between hip and waist measurements (hip-waist) indicated a body shape which visually appeared to be a Triangle, with a smaller bust and larger lower (waist and hip) circumferences (Fig. 2). While these scans may have seemed similar to the Diamond or Oval shape, the larger hip than waist measure indicated an abdomen that was not prominent enough to be considered a Diamond or Oval shape. To capture plus size scans with a large abdomen and a slightly larger hip, a further calculation was added to the Triangle measurement to assess whether the bust was smaller than the waist. After the original formula to determine a Triangle shape, a second formula, if (bust-waist) $<0$, then if (hip-waist) $\geq 0$, captured scans with a larger abdomen and hip compared to the bust. Taking into consideration how plus size women often carry weight in their abdomen as opposed to their hip, this change to the formula more accurately categorized these scans.

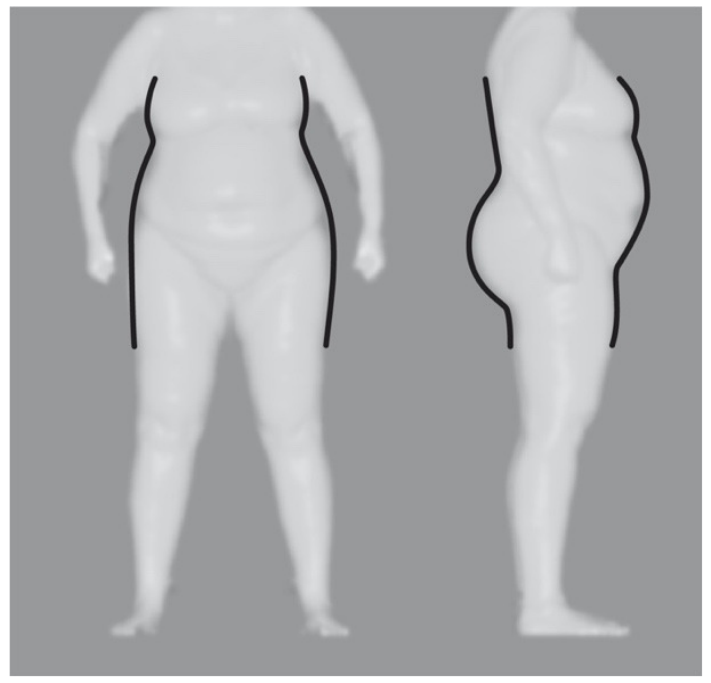

Fig. 2. Subject \#3, whose smaller bust and larger hip circumferences visually indicate a Triangle shape.

\section{Results}

\subsection{Modified formulas}

Table 9 presents the modified FFIT formulas based on the criteria described in the experimental procedures. Table 10 demonstrates how the three subjects' scans in Table 8 would be sorted using the modified formulas. The scan previously sorted as an Inverted Triangle (Fig. 1), but had a large abdomen, is now classified as an Oval. Similarly, Subject \#2 (Fig. 1), which was initially classified as a Rectangle, is now a Diamond, given that the abdomen is greater than bust and hip. Subject \#3 (Fig. 2), which was also a Rectangle with the original formulas, is now classified as a Triangle according to the modified formula which includes scans with a smaller bust than waist and a smaller waist than hip.

Table 9. Modified FFIT formulas (in inches), adapted from Lee et al. [7].

\begin{tabular}{|l|l|}
\hline BODY TYPE & MEASUREMENT \\
\hline Hourglass & If (bust-hip) $\leq 1$, then if (hip-bust) $<3.6$, then if (bust-waist) $\geq 9$ or (hip-waist) $\geq 10$ \\
\hline $\begin{array}{l}\text { Bottom } \\
\text { Hourglass }\end{array}$ & If (hip-bust) $\geq 3.6$ and (hip-bust) $<10$, then if (hip-waist) $\geq 9$, then if (high hip/waist) $<1.193$ \\
\hline Top Hourglass & If (bust-hip) $>1$ and (bust-hip) $<10$, then if (bust-waist) $\geq 9$ \\
\hline Spoon & If (hip-bust) $>2$, then if (hip-waist) $\geq 7$, then if (high hip/waist) $\geq 1.193$ \\
\hline Triangle & If (hip-bust) $\geq 3.6$, then if $0 \leq$ (hip-waist) $<9$, or if (bust-waist) $<0$, then if (hip-waist) $\geq 0$ \\
\hline Inverted Triangle & If (bust-hip) $\geq 3.6$, then if (bust-waist) $<9$, then if (hip-waist) $\geq 0$ \\
\hline Rectangle & $\begin{array}{l}\text { If (hip-bust) }<3.6, \text { and (bust-hip) }<3.6, \text { then if } 0 \leq \text { (bust-waist) }<9 \text { and } 0 \leq \text { (hip-waist) }< \\
10\end{array}$ \\
\hline Diamond & If (hip-waist) $<0$, and (bust-waist) $<0$ \\
\hline Oval & If (hip-waist) $<0$, and (bust-waist) $\geq 0$ \\
\hline
\end{tabular}


Table 10. Size 16 subjects which were incorrectly identified, reclassified with the modified formulas.

\begin{tabular}{|l|l|l|l|l|l|l|l|}
\hline SUB & $\begin{array}{l}\text { BUST- } \\
\text { HIP }\end{array}$ & $\begin{array}{l}\text { HIP- } \\
\text { BUST }\end{array}$ & $\begin{array}{l}\text { BUST- } \\
\text { WAIST }\end{array}$ & $\begin{array}{l}\text { HIP- } \\
\text { WAIST }\end{array}$ & $\begin{array}{l}\text { HIGH HIP- } \\
\text { WAIST }\end{array}$ & $\begin{array}{l}\text { INITIAL BODY } \\
\text { SHAPE }\end{array}$ & $\begin{array}{l}\text { NEW BODY } \\
\text { SHAPE }\end{array}$ \\
\hline$\# 1$ & $4.88 "$ & $-4.88 "$ & $4.17 "$ & $-0.71 "$ & 1.07 & Inverted Triangle & Oval \\
\hline$\# 2$ & $-1.81 "$ & $1.81 "$ & $-3.90 "$ & $-2.09 "$ & 0.99 & Rectangle & Diamond \\
\hline$\# 3$ & $-2.95 "$ & $2.95 "$ & $-2.83 "$ & $0.12 "$ & 1.05 & Rectangle & Triangle \\
\hline
\end{tabular}

\section{Discussion and Conclusions}

\subsection{Why understanding body shape is important}

Taking into consideration how plus-size women can carry weight around their abdomen as opposed to their hips, the modified formulas more accurately categorize the 3D scans. Shape information can inform how manufacturers should design apparel silhouettes, integrate adjustment features, zone materials (stretch and no stretch), fit and grade patterns for plus size women. Commercial apparel products should not just be based on a set of measures, but also through an understanding of how those measures are distributed around a body throughout a size range. Sokolowski and Bettencourt have also found that the same body shape does not consistently exist across a size range [6].

\subsection{Future work}

The researchers are currently working on a study to determine if the shapes defined by the FFIT are culturally relevant. We are living in a time, where equality and body positivity are paramount, but the apparel industry is still using size naming conventions (e.g., plus size, wide, oval) that may need to be updated. The aim of the study will be to educate the apparel industry about appropriate body shape descriptors, as defined by the consumer. As manufacturers strive to create products for every-body and aim for all body types to be treated equally consumers deserve better tools and communication to determine apparel fit.

\section{References}

[1] C. M. Hales, M. D. Carroll, C. D. Fryar, and C. L. Ogden, "Prevalence of obesity among adults and youth: United States 2015-2016", NCHS Data Brief, No.288, 2017.

[2] K. Simmons, C. L. Istook, and P. Devarajan, "Female Figure Identification Technique (FFIT) for apparel part I: Describing female shapes", in Journal of Textile and Apparel, Technology and Management, Vol.4, No.1, 2004.

[3] K. Simmons, C. L. Istook, and P. Devarajan, "Female Figure Identification Technique (FFIT) for apparel part II: Development of shape sorting software", Journal of Textile and Apparel, Technology and Management, Vol.4, No.1, 2004.

[4] H. George-Parkin, "68\% of American Women Wear a Size 14 or Above," Racked, Jun. 5, 2018, https://www.racked.com/2018/6/5/17380662/size-numbers-average-woman-plus-market., accessed Sep. 8, 2019.

[5] S. L. Sokolowski, L. Griffin, J. Silbert, "The Variability of US Women's Plus Size Product Sizing and Self-Identified Size 18 Bodies", in International Conference on Applied Human Factors and Ergonomics, Jul. 24, 2019, pp.124-133, https://doi.org/10.1007/978-3-030-20470-9 15.

[6] S. L. Sokolowski and C. Bettencourt, "How Understanding Female Plus Size Body Shapes Throughout a Size Range Can Affect Apparel Grading and Design Attributes," in Proceedings of the International Textiles and Apparel Association. In Press.

[7] J. Y. Lee, C. L. Istook, Y. J. Nam, and S. M. Park, "Comparison of body shape between USA and Korean women", in International Journal of Clothing Science and Technology, Vol.19, No.5, 2007, pp. 374-391, https://doi.org/10.1108/09556220710819555. 